\title{
Removal of EMG Artifacts from Multichannel EEG Signal Using Automatic Dynamic Segmentation and Adaptive Thresholding with Multilevel Decomposed Wavelets
}

\author{
K.P. Paradeshi ${ }^{1}$, Research Scholar, Professor Dr. U.D. Kolekar ${ }^{2}$, \\ ${ }^{l}$ Associate Professor, PVPIT, Budhgaon Sangli, Maharashtra. \\ ${ }^{2}$ Professor and Principal, APSIT, Thane. Mumbai, Maharashtra.
}

\begin{abstract}
:
Background: Like the brain, muscles also generate electrical signals. These signals are picked up by EEG electrodes and become muscle artifacts in EEG recording. MA is looking like fast oscillations. EEG signals disturbed by MA have larger amplitudes than normal EEG signals. Human beings have a large number of muscles all over their bodies. The muscle movement that happens near electrodes, such as teeth squeezing, jaw clenching, forehead movements will have a huge impact on the power spectrum of EEG signals. Usually, presence of the MA in EEG signals will increase the power of EEG signals in the frequency band from roughly $20 \mathrm{~Hz}$ to $50 \mathrm{~Hz}$.

Materials and methods: 16 channel EEG signals are acquired with EMG artifacts. The subject is instructed to do jaw clenching, forehead movement, teeth squeezing at different instances during the time of recording. The captured EEG signal is imported in MATLAB. Sampling rate used is $1024 \mathrm{~Hz}$. Statistical parameters like PSNR, RMSE are used for comparison. EMG artifacts frequently affected the EEG signal and contaminated valuable information. The present work deals with novel automatic dynamic size independent components based on statistical information of signal and development of multilevel decomposition with adaptive threshold for removing of EMG artifacts. Automatic and dynamic segmentation is the major feature of this method. A particular segment can be analyzed and processed independent of other segments. The present adaptive threshold method is best suitable for removal of muscle artifacts.
\end{abstract}

Results: Present method is better for suppression of EMG artifacts and preserves brain neural activity information as compared with static segmentation.

Conclusion: Automatic dynamic segmentation method with adaptive thresholding of multilevel decomposed wavelets is showing superior performance over conventional static segmentation method. It removes EMG artifacts significantly by preserving brain neural activity.

Keywords: Adaptive threshold, muscle artifacts, Automatic segmentation, RMSE, dynamic partition, PSNR.

\section{Introduction}

The disturbing myogenic activity not only powerfully affects the visual analysis of EEG, but also most certainly impairs the results of EEG signal processing tools such as source localization. This discussion focuses on the particular context of the contamination epileptic signals (interictal spikes) by muscle artifact, as EEG is a key diagnostic tool for this pathology. In this context, the aim was to evaluate the ability of two stochastic approaches of blind source separation, namely independent component analysis (ICA) and canonical correlation analysis (CCA), and of two deterministic approaches, namely empirical mode decomposition (EMD) and wavelet transform (WT) to remove muscle artifacts from EEG signals. To quantitatively compare the performance of these four algorithms, epileptic spike-like EEG signals were simulated from two different source configurations and artificially contaminated with different levels of real EEG-recorded myogenic activity. The efficacy of CCA, ICA, EMD, and WT to correct EMG artifact was evaluated both by calculating the normalized MSE between denoised and original signals and by comparing the results of source localization obtained from an artifact-free as well as raw signals, before and after artifact correction.Tests on real data recorded in an epileptic patient are also presented. The results obtained in the context of simulations and real data show that EMD outperformed the three other algorithms for the denoising of data highly contaminated by muscular activity. For less noisy data, and when spikes arose from a single cortical source, the myogenic artifact was best corrected with CCA and ICA. Otherwise when spikes originated from two distinct sources, either EMD or ICA offered the most reliable denoising result in highly noisy data, while WT offered the better denoising result for less noisy data. These results suggest that the performance of muscle artifact correction methods strongly depends on the level of data contamination, and of the source configuration underlying EEG signals [1].

A novel technique (Automatic Wavelet Independent Component Analysis, AWICA) for automatic EEG artifact removal is presented. AWICA is based on the joint use of the Wavelet Transform and of ICA.It 
consists of a two-step procedure relying on the concepts of kurtosis and Renyi's entropy. Both synthesized and real EEG data are processed by AWICA and the results achieved were compared to the ones obtained by applying to the same data the "wavelet enhanced" ICA method recently proposed by other authors. Simulations illustrate that AWICA compares favorably to the other technique. The method here proposed is shown to yield improved success in terms of suppression of artifact components while reducing the loss of residual informative data, since the components related to relevant EEG activity are mostly preserved [2].

A new method for muscle artifact removal in EEG is presented, based on canonical correlation analysis (CCA) as a blind source separation (BSS) technique. This method is demonstrated on a synthetic data set. The method outperformed a low-pass filter with different cutoff frequencies and an independent component analysis (ICA) based technique for muscle artifact removal. In addition, the method is applied to a real ictal EEG recording contaminated with muscle artifacts. The proposed method removed successfully the muscle artifact without altering the recorded underlying ictal activity $[3,7]$.

The methods of wavelet threshold denoising and independent component analysis (ICA) are introduced. ICA is a novel signal processing technique based on high order statistics, and is used to separate independent components from measurements. The extended ICA algorithm does not need to calculate the higher order statistics, converges fast, and can be used to separate subgaussian and supergaussain sources. A prewhitening procedure is performed to decorrelate the mixed signals before extracting sources. The experimental results indicate the electromyograpm (EMG) and electrocardiograh (ECG) artifacts in electroencephalograph (EEG) can be removed by a combination of wavelet threshold denoising and ICA [4].

The electroencephalogram (EEG) signals were usually contaminated by electromyography (EMG) signals. Multivariate empirical mode decomposition (MEMD) based method is proposed to remove EMG artifacts from the EEG signals. Firstly, the EEG signals were decomposed by the MEMD into multiple multivariate intrinsic mode functions (MIMFs) with different frequency bands. Then the power spectra were calculated for every MIMF by using the Welch method. Because the power spectrum of EEG and EMG were focused on different frequency ranges, the MIMFs which included the EMG artifacts could be got rid of. Finally, the clean EEG could be reconstructed by the remaining MIMFs. In this study, the MEMD-based method was used to remove the EMG artifacts for different signal-to-noise ratio (SNR). The experimental results indicated that the SNR of EEG signals could be obviously improved in different conditions and the mean square error (MSE) of EEG signals also could be significantly reduced [5].

The artifacts appear as noise in the recorded EEG signal individually or in a combined manner. Usually physicians are misled by these noisy signals and the EEG analysis can go wrong. Removal of noise signal which can be EMG, ECG or a combination of these two artifacts from the corrupted EEG signal and also signal enhancement both using recurrent learning technique are carried out. For this purpose RTRL (Real Time Recurrent Learning) algorithm is implemented which is the most recent and sophisticated real time neural network algorithm [6].

\section{Methodology}

16 channel EEG signals are acquired with EMG artifacts. The subject is instructed to do jaw clenching, forehead movement, teeth squeezing at different instances during the time of recording. The captured EEG signal is imported in MATLAB. Sampling rate used is $1024 \mathrm{~Hz}$. Statistical parameters like Standard deviation, PSNR, RMSE are used for comparison. EMG artifacts frequently affected the EEG signal and contaminated valuable information. Dynamic segmentation is carried out to partition the EEG signal into a number of independent components. Segments are unequal length and automatic segmentation is done using the property of stationary waves, hence, called as dynamic segments. EEG signal is non-stationary but within segments, each IC has unique stationary properties. Global threshold value (GTV) of raw EEG is calculated using the property of mean, median and standard deviation. Out of these threshold values the numerical maximum selected as GTV.

Two levels Discrete Wavelet Transform (DWT) of each IC is taken using 'HAAR' wavelet family. One IC is first decomposed into four wavelets. It computes the approximation coefficients matrix cA and details coefficients matrices $\mathrm{cH}, \mathrm{cV}$, and $\mathrm{cD}$ (horizontal, vertical, and diagonal, respectively), obtained by wavelet decomposition of the input matrix .Successive DWT is taken of the decomposed wavelets. Thus, the wavelets are distributed as 4 wavelets for first decomposition, 16 wavelets for second decomposition, 64 wavelets for third decomposition, 256 wavelets for fourth decomposition, and 1024 wavelets for fifth decomposition. This process of $\mathrm{n}^{\text {th }}$ decomposition is restricted to size of RAM memory and processing power of central processing unit. Hence, 3 to 5 decomposition is sufficient to greater accuracy. $n=1$ is the normal wavelet based method.

Local Threshold Value (LTV) is calculated using mean, median and standard deviation of decomposed wavelets. Numerical maximum out of these selected as LTV. Total number of LTV is equal to number of wavelets 'M'. GTV and LTV are relative to the DWT method as decomposition progressed, previously calculated LTV treated as GTV in the next decomposition. Threshold means replacement of the values in the 
wavelets greater than LTV with new value ' $\varepsilon$ '. It is categorised depending on the value of ' $\varepsilon$ '. $\varepsilon=0, \mu$, GTV and spatial called respectively as zero replacement threshold, mean replacement threshold, maximum level threshold and co-ordinate to co-ordinate spatial replacement threshold in this literature. Zero replacement threshold removes all information where as it is assumed to be the original information lies in between mean and GTV. Threshold is carried out at highest (suppose $\mathrm{n}=5$, at $5^{\text {th }}$ decomposition) decomposed wavelets and after completion of process; Inverse DWT (IDWT) is taken whenever move to lower decomposition. Spatial threshold is implemented as progressed to lower decomposed ( $n=5$ to 4,4 to 3,3 to 2 and 2 to 1 decomposition) wavelets. At the $1^{\text {st }}$ decomposition, it is replaced with spatial data which is better estimation of original data in the artifact zone. Final clean EEG is obtained by IDWT of first decomposed wavelet.

\section{Dynamic Segmentation}

EEG is non-stationary signal which does not possess certain properties of statistics. A stationary signal is significant in the signal processing due to its predefined statistical property. As EEG is non-stationary, it is partitioned into number of segments in which within that segments signal possess stationary property. These segments are treated as independent components of EEG signal. Analysis and processing of EEG segments provides noise filtering, artifacts removing and many more applications. A segment is valid, if it satisfies law of statistics for stationary signal. Analysis of non-stationary segments results into uncorrelated data and loss of valuable certain information within that segment. Equal size segments do not hold property of stationary signal. Equal size static segmentation method is not suitable for EEG signal processing. Hence, unequal size or dynamic segmentation is desirable.

Nonstationarity of signal can be quantified on the regular time lags by measuring statistics of the signal. The signal within time lag deemed as stationary if there is no considerable variation in these statistics. Skewness, Kurtosis and discriminate are the measure statistical property of time lag provides the information regarding signal. These parameters are vital in the determination of segments and artifacts present in the signal. Skewness is a measure of symmetry or, more precisely, the lack of symmetry of the distribution. A distribution, or data set, is symmetric if it looks the same to the left and right of the center point. The skewness is defined for a real signal as,

$$
\text { Skewness }=\frac{E\left[(x(n)-\mu)^{3}\right]}{\sigma^{3}}
$$

Algorithm for automatic segmentation is as follows,

I: Take channel as counter. Initially select first channel.

II: obtain number of Rows $(\mathrm{R})$ and column $(\mathrm{C})$ of channel.

III: Initialize a variable to store RowStart of each segment.

IV: Take maximum row as counter

V: calculate skewness and calculate difference of current and old values of skewness.

VI: if difference is greater than 0.5 then mark the current row as end of segment and (row +1$)$ as start of new segment. Store the values of skewness as old segment values.

VII: repeat V to VI to last row.

VIII: store the RowStart to the memory

IX: repeat II to VIII to last channel.

Wavelet threshold process is used to remove EMG artifacts present in the EEG signal. Threshold means replacing current data which is greater than threshold value with the new value. Threshold value is calculated as,

$\gamma 1=\frac{N}{\mu+\sigma}$

$\gamma 2=\operatorname{mad} * 1.5$

$\mathrm{N}=100$, mad $=$ median absolute deviation of signal

$\gamma=\max (\gamma 1, \gamma 2)$

Threshold value is the numerical maximum of $\gamma 1, \gamma 2$.

Selection of the threshold value $\gamma$ is an essential element of the algorithm. Here simple fixed form threshold can be used.

$\gamma=\sqrt{2 \log N} \sigma \quad$ (5)

where $N$ is the length of the data segment to be processed, and $\sigma^{2}=$ median $(|W(d, b)|) / 0.6745$ is the estimator of the magnitude of the neural wide band signal part. 


\section{Results And Discussion}

Mainly three different types of artifacts are considered for analysis. Table 1 shows the values of PSNR and RMSE of Subject 1 and Subject 2 due to jaw clenching artifact. Table 2 shows the values of PSNR and RMSE of Subject 3 and Subject 4 due to teeth squeezing artifact. Table 3 shows the values of PSNR and RMSE of Subject 5 and Subject 6 due to forehead movement artifact. Figure 1 shows the raw EEG signal with jaw clenching artefact and Figure 2 shows the clean EEG signal after removal of jaw clenching artifact. Figure 3 shows the raw EEG signal with teeth squeezing artifact and Figure 4 shows the clean EEG signal after removal of teeth squeezing artifact. Figure 5 shows the raw EEG signal with forehead movement artifact and Figure 6 shows the clean EEG signal after removal of forehead movement artifact. For Subject 1 PSNR value is 3.16 whereas RMSE is 25.71 for jaw clenching artifacts. Actually statistically results are dependent upon how heavily artifacts are buried in the signal. Automatic dynamic segmentation method is powerful new technique for removal of EMG artifacts. Clearly we can visualize the correction made by this method for removal of different EMG artifacts as shown in Figure 1 to Figure 6.

Table 1: Results of EMG artifact due to Jaw Clenching

\begin{tabular}{|l|l|l|}
\hline Subject(s) & PSNR & RMSE \\
\hline Subject 1 & 3.16 & 25.71 \\
\hline Subject 2 & 3.862 & 22.77 \\
\hline
\end{tabular}

Figure 1: Raw EEG signal with jaw clenching EMG artifacts Subject1

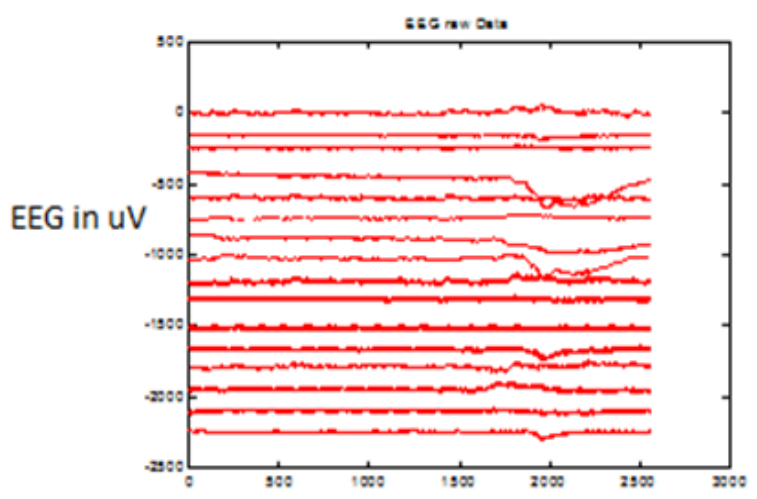

Time in sec

Figure 2: Clean EEG signal after removal of jaw clenching EMG artifacts of Subject 1

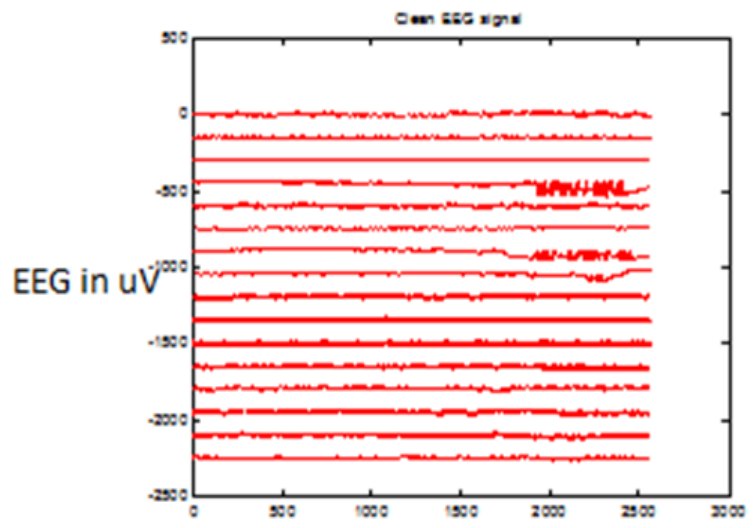

Time in sec

Table 2: Results of EMG artifacts due to teeth squeezing

\begin{tabular}{|l|l|l|}
\hline Subject(s) & PSNR & RMSE \\
\hline Subject 3 & 7.8767 & 16.99 \\
\hline Subject 4 & 5.6916 & 17.50 \\
\hline
\end{tabular}


Figure 3: Raw EEG signal with teeth squeezing EMG artifacts of Subject 3

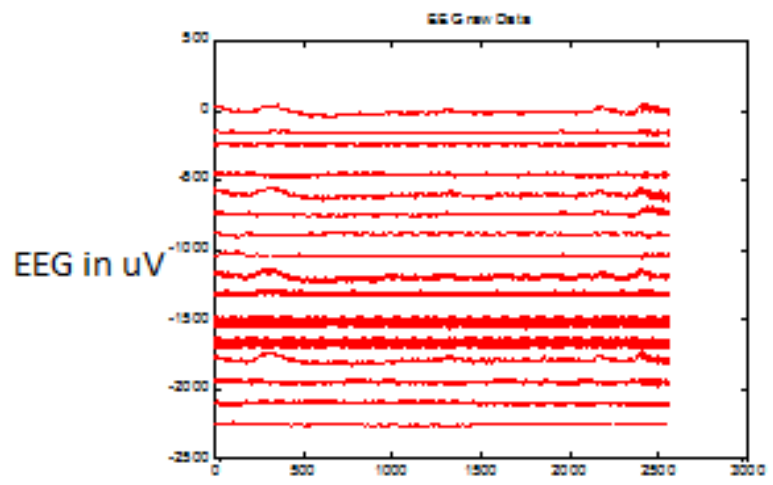

Time in sec

Figure 4: Clean EEG signal after removal of teeth squeezing EMG artifacts of Subject 3

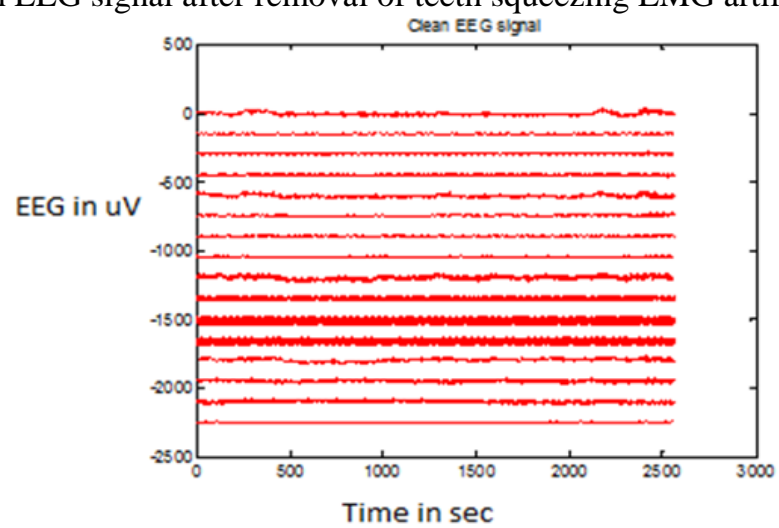

Table 3: Results of EMG artifacts due to forehead movements

\begin{tabular}{|l|l|l|}
\hline Subject(s) & PSNR & RMSE \\
\hline Subject 5 & 11.9759 & 24.99 \\
\hline Subject 6 & 11.1842 & 22.69 \\
\hline
\end{tabular}

Figure 5: Raw EEG signal with forehead movement EMG artifact of Subject 5

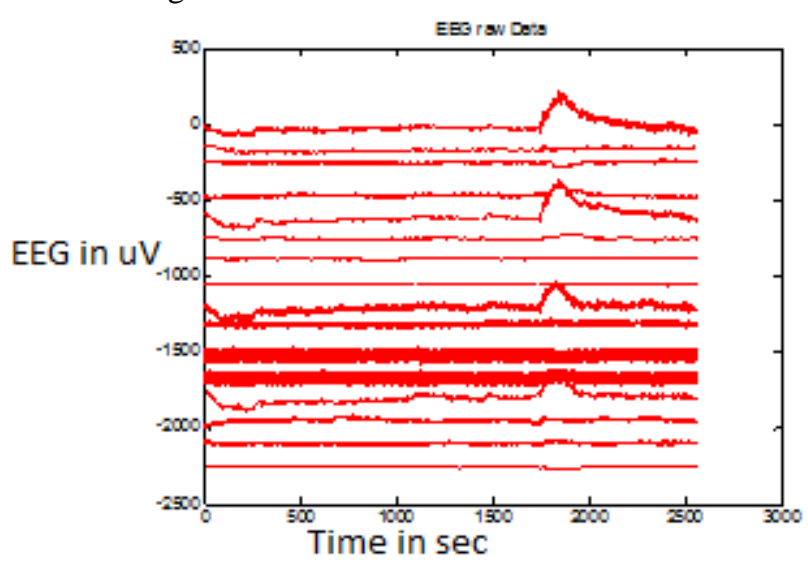

Figure 6 Clean EEG signal after removal of forehead movement EMG artifact of Subject 5 


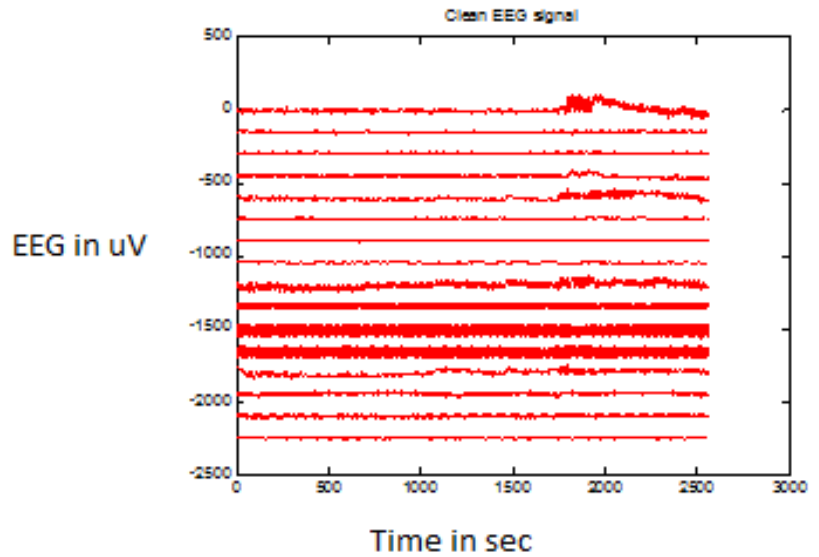

Table 4 shows the results of PSD and Standard Deviation of raw EEG signal and clean EEG signal for different types of EMG artifacts. These performance parameters are used for analysis of different types of artifacts. Mainly Subject 1 and Subject 2 are showing the vales for jaw clenching EMG artifacts. For Subject 1 PSD of Raw EEG signal is 17.468 whereas PSD of clean EEG signal is 13.5021.Similarly Standard deviation values are 17.3772 for raw EEG signal and 6.0837 for clean EEG signal are tabulated. Subject 3 and Subject 4 are concerned with Teeth squeezing artifacts and their values are also tabulated in table 4.Forehead movement EMG artifacts is concerned with Subject 5 and Subject 6. Raw EEG signals are having higher values of PSD and standard deviation whereas clean EEG signals are having lower values of PSD and standard deviation. We have also obtained the minimum values of RMSE.

Table 4 Results of PSD and Standard Deviation of raw EEG signal and clean EEG signal.

\begin{tabular}{|l|l|l|l|l|l|}
\hline Subject(s) & Type of EMG Artifacts & PSD of Raw EEG in dB & $\begin{array}{l}\text { PSD of Clean EEG in } \\
\text { dB }\end{array}$ & $\begin{array}{l}\text { Standard Deviation } \\
\text { of Raw EEG }\end{array}$ & $\begin{array}{l}\text { Standard Deviation } \\
\text { of Raw EEG }\end{array}$ \\
\hline Subject 1 & Jaw clenching & 17.468 & 13.5021 & 17.3772 & 6.0837 \\
\hline Subject 2 & Jaw clenching & 22.7844 & 19.4531 & 9.7918 & 3.4885 \\
\hline Subject 3 & Teeth squeezing & 21.3917 & 14.7326 & 6.1659 & 5.8379 \\
\hline Subject 4 & Teeth squeezing & 22.2516 & 17.4126 & 6.9151 & 5.44 \\
\hline Subject 5 & Forehead Movement & 29.2146 & 22.5581 & 17.2508 & 8.4881 \\
\hline Subject 6 & Forehead Movement & 26.6826 & 21.76 & 15.1939 & 8.0253 \\
\hline
\end{tabular}

\section{Conclusion}

Results obtained using present method is more promising as compared to earlier methods. Lower values of RMSE and higher values of PSNR means artifacts are suppressed predominantly. Automatic dynamic segmentation and adaptive thresholding with multilevel decomposed wavelets is best suited for removal of different types of EMG artifacts. The said method is showing superior performance over conventional static segmentation method. Raw EEG signals are having higher values of PSD and standard deviation whereas clean EEG signals are having lower values of PSD and standard deviation. It indicates that after applying the said techniques different types of EMG artifacts mentioned in the study are greatly suppressed.

\section{References}

[1]. Safieddine et al. EURASIP Journal on Advances in Signal Processing 2012, 2012:127.

[2]. Nadia Mammone, Fabio La Foresta, Carlo Morabito, Automatic Artifact Rejection From Multichannel Scalp EEG by Wavelet ICA, IEEE SENSORS JOURNAL, VOL. 12, NO. 3 MARCH 2012.

[3]. Wim De Clercq, Anneleen Vergult, Bart Vanrumste, Wim Van Paesschen, Sabine Van Huffel, Canonical Correlation Analysis Applied to Remove Muscle Artifacts From the Electroencephalogram, IEEE TRANSACTIONS ON BIOMEDICAL ENGINEERING,2006.

[4]. Weidong Zhou, Jean Gotman, Removal of EMG and ECG Artifacts from EEG Based on Wavelet Transform and ICA, Proceedings of the 26th Annual International Conference of the IEEE EMBS San Francisco, CA, USA September 1-5, 2004.

[5]. Chaolin Teng, Yanyan Zhang, Gang Wang, The Removal of EMG Artifact from EEG Signals by the Multivariate Empirical Mode decomposition, 978-1-4799-5274-8/14/2014 IEEE.

[6]. H. N. Suresh, C. Puttamadappa, Removal OF EMG and ECG artifacts from EEG based on real time recurrent learning algorithm, International Journal of Physical Sciences Vol. 3 (5), pp. 120-125, May, 2008.

[7]. Xun Chen, Chen He, Hu Peng, Removal of Muscle Artifacts from Single-Channel EEG Based on Ensemble Empirical Mode Decomposition and Multiset Canonical Correlation Analysis, Journal of Applied Mathematics, Hindawi Publishing Corporation, Volume 2014, Article ID 261347, 10 pages, http://dx.doi.org/10.1155/2014/261347. 